\title{
Constructal multi scale cylinders with rotation cooled by natural convection
}

\author{
L.G. Page ${ }^{\mathrm{a}}$, T. Bello-Ochende ${ }^{\mathrm{a}, *}$, J.P. Meyer ${ }^{\mathrm{a}}$ \\ ${ }^{a}$ Department of Mechanical and Aeronautical Engineering, University of Pretoria, Pretoria, Private Bag X20, Hatfield, 0028, South Africa
}

\begin{abstract}
In this paper we investigated the thermal behaviour of an assembly of multi scale cylinders in a staggered counterrotating configuration cooled by natural convection with the objective of maximizing the heat transfer density rate (heat transfer rate per unit volume). A numerical model was used to solve the governing equations that describe the temperature and flow fields and a mathematical optimisation algorithm was used to find the optimal structure for flow configurations with two degrees of freedom. The multi scale structure of the cylinder assembly was optimized for each flow regime (Rayleigh number) and cylinder rotation speed for two degrees of freedom. Smaller cylinders were placed at the entrance to the assembly, in the wedge-shaped flow regions occupied by fluid that had not yet been used for heat transfer, to create additional length scales to the flow configuration.

It was found that there was almost no effect of cylinder rotation on the maximum heat transfer density rate, when compared to stationary cylinders, at each Rayleigh number; with the exception of high cylinder rotation speeds, which served to suppress the heat transfer density rate. It was, however, found that the optimized spacing decreased as the cylinder rotation speed was increased at each Rayleigh number. Results further show that the maximum heat transfer density rate for a multi scale configuration (without cylinder rotation) was higher than a single scale configuration (with rotating cylinders) with an exception at very low Rayleigh numbers.
\end{abstract}

Keywords: Natural convection, Rotating cylinders, Heat transfer density rate, Counter-rotation, Optimal packing, Multi scale, Mathematical Optimization

\section{Introduction}

Efficiency is a key aspect in design, which has become prevalent in the design of heat transfer devices such as heat sinks and pin fins. Research has been and is still being conducted on this subject with the aim of extracting more and more heat from a given space through the maximizing of the packing of heat-generating material per unit volume. This drive to augment heat transfer devices has become reinforced by modern electronic systems which produce high amounts of heat due to the ever increasing power-to-volume ratio employed in such systems.

The strive for greater heat transfer density rates has been the driving force behind many of the miniaturization efforts, augmentations and unconventional ways of designing heat transfer devices. This has lead researchers to study the optimized configurations for various architectures such as: the optimal spacing of parallel plates in forced convection, natural convection and mixed convection $[7,8,10,35]$; the optimal spacing of cylinders in forced convection and natural convection [36, 37]; and various optimized multi-scale structures [4, 9, 11-13, 34], etc.

The heat transfer and fluid flow around a single rotating cylinder has been studied previously. Badr and Dennis [1] considered the problem of laminar forced convective heat transfer from an isothermal circular cylinder rotating about its own axis located in a uniform stream. The authors reported that the temperature fields are strongly influenced by the rotational speed of the cylinder and contradictory to expectation they found that the overall heat transfer coefficient tends to decrease as the rotational of the cylinder increases. They attributed this to the presences of a rotating fluid layer around the cylinder that separates the cylinder from the main flow stream.

\footnotetext{
${ }^{*}$ Corresponding author

Email address: Tunde.Bello-Ochende@up.ac.za (T. Bello-Ochende )
} 


\begin{tabular}{|c|c|c|c|}
\hline \multicolumn{4}{|c|}{ Nomenclature } \\
\hline$D_{0}$ & large cylinder diameter $(\mathrm{m})$ & \multicolumn{2}{|c|}{ Greek symbols } \\
\hline$f(\mathbf{x})$ & objective function & $\alpha$ & thermal diffusivity $\left(\mathrm{m}^{2} / \mathrm{s}\right)$ \\
\hline g & gravitational acceleration $\left(\mathrm{m} / \mathrm{s}^{2}\right)$ & $\beta$ & thermal expansion coefficient $(1 / K)$ \\
\hline$g(\mathbf{x})$ & inequality constraint function & $\mu$ & viscosity $(\mathrm{kg} / \mathrm{ms})$ \\
\hline$H_{d}$ & downstream flow length (m) & $\delta_{T}$ & thermal boundary layer thickness (m) \\
\hline$H_{u}$ & upstream flow length (m) & $v$ & kinematic viscosity $\left(\mathrm{m}^{2} / \mathrm{s}\right)$ \\
\hline$k$ & thermal conductivity $(\mathrm{W} / \mathrm{mK})$ & $\rho_{0}$ & reference fluid density $\left(\mathrm{kg} / \mathrm{m}^{3}\right)$ \\
\hline$W$ & assembly length $(\mathrm{m})$ & $\omega_{0}$ & cylinder angular velocity $(\mathrm{rad} / \mathrm{s})$ \\
\hline$P$ & pressure $(\mathrm{Pa})$ & & \\
\hline $\operatorname{Pr}$ & Prandtl number & \multicolumn{2}{|c|}{ Subscripts } \\
\hline$\tilde{q}$ & dimensionless heat transfer density rate & $m$ & maximum \\
\hline$q^{\prime}$ & heat transfer rate per unit length $(\mathrm{W} / \mathrm{m})$ & opt & optimum \\
\hline$q^{\prime \prime \prime}$ & heat transfer density rate $\left(\mathrm{W} / \mathrm{m}^{3}\right)$ & & \\
\hline$R a$ & Rayleigh number & \multicolumn{2}{|c|}{ Superscripts } \\
\hline$S_{0}$ & spacing between large cylinders (m) & * & optimal design variable \\
\hline$T$ & fluid temperature $(\mathrm{K})$ & & \\
\hline$T_{w}$ & wall temperature $(\mathrm{K})$ & Accents & \\
\hline & inlet fluid temperature $(\mathrm{K})$ & $\approx$ & dimensionless variable \\
\hline $\mathbf{U}$ & fluid velocity vector $(\mathrm{m} / \mathrm{s})$ & $\hat{r}$ & unit vector \\
\hline$(u, v, w)$ & fluid velocity components (m/s) & & \\
\hline & optimisation design variables & & \\
\hline$(x, y, z)$ & Cartesian coordinates $(\mathrm{m})$ & & \\
\hline
\end{tabular}

Chiou and Lee [15] considered a problem of forced convection on a rotating cylinder cooled with an air jet. The results confirmed that the overall heat transfer is enhanced at lower rotational speeds and at higher rotational speeds the effect became reversed. They attributed this to the presences of a layer of dead air around the cylinder.

Panda and Chhabra [30] considered a problem of forced convection heat transfer from a heated cylinder rotating in streaming power-law fluids. The results show a similar behaviour of the heat transfer rate: for moderate rotational velocities at low Reynolds numbers the heat transfer rate is enhanced and there is an envelope of conditions (Reynolds number, rotation speed and power-law index) in which rotation has a negative effect on the heat transfer rate. Similar research includes the works of Gshwendtner [17], Mohanty et al. [23], Oesterle et al. [25], Ozerdem [28], Paramane and Sharma [31, 32], Yan and $\mathrm{Zu}$ [38] and Nobari et al. [24],

More recent studies have been conducted by Joucaviel et al. [20], with a single scale structure of a row of heatgenerating rotating cylinders cooled by forced convection. The authors reported that the effect of rotation was beneficial from the point of view of maximising the heat transfer density rate. The results also showed that a counter-rotation configuration increases the heat transfer density rate more efficiently when compared to a co-rotation configuration. In fact the authors states that a co-rotating configuration "seems to be useless", in that the heat transfer density rate decreases and the optimal spacing increases with the increase in rotational speed. Similarly, Bello-Ochende et al. [14] built onto the work by Bello-Ochende and Bejan [11] by considering a multi-scale constructal design with rotating cylinders.

The study presented in this paper builds onto prior research by Bello-Ochende and Bejan [12], in which the authors optimized the cylinder-to-cylinder spacings in a multi-scale constructal design of heat-generating cylinders (without cylinder rotation) cooled by natural convection for one and two degrees of freedom. These classical results will be used as a reference (benchmark) for the results reported in this paper.

It is the purpose of this paper to maximize the heat transfer density rate of a multi scale configuration of heatgenerating rotating cylinders in steady laminar single-phase natural convection, through constructal theory and design $[2,3,5,6]$. According to this method, the flow configuration is free to morph (change) in the pursuit of maximising global performance subject to global constraints. The resulting optimal configuration is determined.

The assembly of cylinders rotate in a staggered counter-rotating configuration, with stationary small diameter 
cylinders and counter-rotating large diameter cylinders. The results for a single row of heat-generating rotating cylinders in natural convection was presented previously by Page et al. [29].

This paper focuses on taking the constructal design of optimal spacings in a new direction by numerically formulating the problem and mathematically optimising the flow configuration. An optimized single scale and multi scale flow structure (subject to global constraints), that achieves even higher levels of heat transfer density rate, is sought for each flow regime (Rayleigh number and cylinder rotation speed). The process of optimising the flow configuration is often one of "brute force", whereby the design space is broken up into a finite number of points at which the global objective is evaluated. By introducing a mathematical optimization routine, a seemingly infinite design space can be evaluated in order to obtain the optimal flow configuration.

\section{Numerical Model}

Consider a row of infinity long, rotating and heat-generating cylinders as shown in Fig. 1. The large diameter cylinders $\left(D_{0}\right)$ are aligned along their centreline and smaller cylinders (diameter $D_{1}$ ) are inserted in the entrance (converging) region of the channels formed between the larger diameter cylinders to form a stacking.

The large diameter cylinders rotate at an angular velocity of $\omega_{0}$ in a staggered counter-rotating configuration. The large cylinder diameter $\left(D_{0}\right)$ is fixed and the surface temperature of all the cylinders $\left(T_{w}\right)$ is assumed uniform and constant and greater than that of the fluid temperature $\left(T_{\infty}\right)$. The cylinders are cooled by natural convection. The objective is to select the number of cylinders (large and small) in the stacking or in other words to select the cylinderto-cylinder spacing $\left(S_{0}\right)$ and the small cylinder diameter $\left(D_{1}\right)$ in such a manner that the overall thermal heat transfer between the cylinders and the ambient air is maximized. This is done for each flow regime (Rayleigh number) and cylinder rotation speed. The smaller cylinders are kept stationary and have a uniform and constant surface temperature $\left(\tilde{T}_{w}\right)$ equal to the surface temperature of the larger cylinders.

The flow is assumed steady, laminar, incompressible and two-dimensional. All thermophysical properties are assumed constant. The temperature variations are assumed sufficiently small relative to the absolute temperature so that the Boussinesq approximation is valid.

The effect of increased complexity is shown in Fig. 2, in which a smaller cylinder (of diameter $D_{1}$ ) is inserted in the entrance (converging) region of the channels formed between the larger cylinders. This geometric change adds one more degree of freedom to the system: the smaller cylinder diameter $\tilde{D}_{1}=D_{0} / D_{1}$. The flow configuration now has two degrees of freedom, represented by the small cylinder diameter $\tilde{D}_{1}$ and the original cylinder-to-cylinder spacing $\tilde{S}_{0}$.

Figure 3 shows the elemental volume that characterises this assembly. The computational domain contains the upstream section $\left[H_{u} \times 2\left(D_{0}+S_{0}\right)\right]$, the downstream section $\left[H_{d} \times 2\left(D_{0}+S_{0}\right)\right]$ and the flow region $\left[D_{0} \times 2\left(D_{0}+S_{0}\right)\right]$. The upstream lengths $\left(H_{u}\right)$ and downstream lengths $\left(H_{u}\right)$ were selected based on mesh independence tests described later in Section 3.

The conservation equations (in vector form) for mass, momentum and energy are respectively:

$$
\operatorname{div}(\mathbf{U})=0
$$

$$
\rho_{o} \frac{D \mathbf{U}}{D t}=-\nabla P+\mu \nabla^{2} \mathbf{U}-\rho_{o} \mathbf{g} \beta\left(T-T_{\infty}\right)
$$

$$
\frac{D T}{D t}=+\alpha \nabla^{2} T
$$

where $\mathbf{U}=[u, v, w]$ is the velocity field and $\nabla^{2}=\partial^{2} / \partial x^{2}+\partial^{2} / \partial y^{2}+\partial^{2} / \partial z^{2}$

The system of coordinates $(x, y)$ and the velocity component $(u, v)$ are defined in Fig. 3 . The variables are defined in the nomenclature. The numerical work of solving Eqs. 1 to 3 is based on dimensionless formulation using the following variables:

$$
\tilde{x}, \tilde{y}, \tilde{y}=\frac{(x, y, z)}{D_{0}}, \quad \tilde{u}, \tilde{v}, \tilde{w}=\frac{(u, v, w)}{\left(\alpha / D_{0}\right)\left(R a_{D_{0}} P r\right)^{1 / 2}}
$$




$$
\begin{aligned}
& \tilde{T}=\frac{T-T_{\infty}}{T_{w}-T_{\infty}}, \quad \tilde{P}=\frac{P}{\left(\alpha \mu / D_{0}^{2}\right)\left(R a_{D_{0}} P r\right)^{1 / 2}} \\
& \tilde{\omega}_{0}=\frac{\omega_{0}}{\left(2 \alpha / D_{0}{ }^{2}\right)\left(R a_{D_{0}} P r\right)^{1 / 2}}
\end{aligned}
$$

where the large cylinder diameter $\left(D_{0}\right)$ is selected as the characteristic length, $\operatorname{Pr}=v / \alpha$ is the Prandtl number and the Rayleigh number is defined in terms of the large cylinder diameter as:

$$
R a_{D_{0}}=\frac{g \beta\left(T_{w}-T_{\infty}\right) D_{0}{ }^{3}}{\alpha v}
$$

Substituting Equations 4 to 7 into Equations 1 to 3 yields the dimensionless form of the continuity, momentum and energy equations respectively:

$$
\begin{aligned}
& \operatorname{div} \tilde{\mathbf{U}}=0 \\
& \left(\frac{R a}{P r}\right)^{1 / 2} \frac{D \tilde{\mathbf{U}}}{D \tilde{t}}=-\tilde{\nabla} \tilde{P}+\tilde{\nabla}^{2} \tilde{\mathbf{U}}+\left(\frac{R a}{P r}\right)^{1 / 2} \tilde{T} \hat{k} \\
& (\operatorname{RaPr})^{1 / 2} \frac{D \tilde{T}}{D \tilde{t}}=+\tilde{\nabla}^{2} \tilde{T}
\end{aligned}
$$

where $\tilde{\mathbf{U}}=[\tilde{u}, \tilde{v}, \tilde{w}]$ is the dimensionless velocity field and $\hat{k}=\left[\begin{array}{lll}0 & 1 & 0\end{array}\right]$ is a unit vector indicating the directions in which gravity acts. All geometric dimensions (cylinder diameters, cylinder-to-cylinder spacing, upstream and downstream lengths) of the computational domain shown in Fig. 3, were also made dimensionless by dividing through by the length scale $D_{0}$ :

$$
\tilde{D}_{0}=\frac{D_{0}}{D_{0}}=1, \quad \tilde{S}_{0}=\frac{S_{0}}{D_{0}}, \quad \tilde{H}_{d}, \tilde{H}_{u}=\frac{H_{d}, H_{u}}{D_{0}}
$$

The computational domain boundary conditions are indicated in Fig. 3. For all the cylinder surfaces, the boundary conditions are specified as zero slip, zero penetration, constant and uniform surface temperature $\left(\tilde{T}_{w}=1\right)$ and the large cylinder surfaces have an angular velocity of $\tilde{\omega}_{0}$. For the inlet of the computational domain, the boundary conditions are specified as $\tilde{P}=0, \tilde{T}_{\infty}=0$ and $\tilde{u}=\partial \tilde{v} / \partial \tilde{y}=0$. For the exit of the computational domain, the boundary conditions are specified as $\partial(\tilde{u}, \tilde{v}) / \partial \tilde{y}=0, \tilde{P}=0$ and $\partial \tilde{T} / \partial \tilde{y}=0$. For the upstream section $\left(0 \leq \tilde{y} \leq \tilde{H}_{u}\right)$ of the computational domain, the boundary conditions are specified as symmetry planes or free slip and no penetration $(\tilde{u}=\partial(\tilde{v}, \tilde{T}) / \partial \tilde{x}=0)$.

For the downstream section $\left(\tilde{H}_{u} \leq \tilde{y} \leq \tilde{H}_{u}+\tilde{D}_{0}+\tilde{H}_{d}\right)$ of the computational domain, two boundary conditions are specified: symmetry plane or free slip and no penetration $(\tilde{u}=\partial(\tilde{v}, \tilde{T}) / \partial \tilde{x}=0)$ at the left side of the flow region and; zero stress $(\partial \tilde{P} / \partial \tilde{x}$ and $\partial \tilde{u} / \partial \tilde{x}=\partial(\tilde{v}, \tilde{T}) / \partial \tilde{x}=0)$ on the right side of the flow region. By specifying $\partial \tilde{u} / \partial \tilde{x}$ on the right side of the flow region, fluid is allowed to flow horizontally into the computational domain. This entrainment effect nullifies the unrealistic vertical acceleration or chimney effect that would have been generated had we specified zero slip on this side.

The cylinder-to-cylinder spacing and the small cylinder diameter is varied and thus we are interested in the geometric configuration that maximizes the overall heat transfer between the cylinders and the surrounding fluid. The dimensionless quantity used to evaluate this configuration is the dimensionless heat transfer density rate. The heat transfer density rate is $q^{\prime \prime \prime}=q^{\prime} / 2 D_{0}\left(D_{0}+S_{0}\right)$, where $q^{\prime}$ is the sum of the total heat transfer rate integrated over the surface of the cylinders:

$$
q^{\prime}=\sum_{i=1}^{d i} \frac{D_{0}}{2} \int_{0}^{2 \pi} k(\nabla T)_{s n} \mathrm{~d} \theta+\sum_{i=1}^{d j} \frac{D_{1}}{a} \int_{0}^{2 \pi} k(\nabla T)_{s n} \mathrm{~d} \theta
$$


where $d i$ is the number of large diameter cylinders, $d j$ is the number of small diameter cylinders, $a=2$ or $a=4$ for a complete and half cylinder respectively, and the subscript $s n$ denotes that gradient of $T$ is taken with respects to the normal direction to the cylinder surface. The corresponding dimensionless heat transfer density rate is:

$$
\tilde{q}=\frac{q^{\prime}}{2 D_{0}\left(D_{0}+S_{0}\right) k\left(T_{w}+T_{\infty}\right)}
$$

\section{Numerical Methods}

Almost all CFD packages or codes are designed with the goal in mind that the CFD package will be used once off for a given engineering problem with maybe a handful of variations in either the geometry or fluid properties being simulated. For an optimisation type problem this "one-by-one set up and solution" method can become very cumbersome as the number of simulations required to be set up and solved becomes exponentially greater with an increased number of design variables. A more efficient method is sought and is presented in this section.

Equations 8 to 10 were solved using a finite volume package (OpenFoam [26]), with hexahedron elements. The velocity-pressure coupled equations were solved using the Pressure-Implicit with Splitting of Operators (PISO) procedure, proposed by Issa [18].

The solution routine of solving the governing equations (8 to 10) with OpenFoam, for each geometric configuration, was automated (as shown in Fig. 4) through the use of a programming script [33] and a few numerical tools $[16,19]$. This automated routine was then coupled to a mathematical optimisation algorithm [19], as shown in Fig. 5, in order to efficiently optimise a design type problem with multiple design variables, subject to global constraints, for a global objective. The optimisation algorithm used in this paper is based on the original algorithm implemented by Kraft [21] which utilises the slightly modified Non-Negative Least Squares (NNLS) algorithm of Lawson [22].

Thus the constrained optimisation problem (derived from Fig. 3 and Eq. 13) can formally be written as:

$$
\begin{aligned}
& \underset{w \text {.r.t. } \mathbf{x}}{\operatorname{minimise}}(\mathbf{x})=-\tilde{q}(\mathbf{x}), \quad \mathbf{x}=\left[x_{1}, x_{2}\right]^{T} \in \mathbb{R}^{n} \\
& \text { such that: } \\
& \qquad \begin{aligned}
g_{2}(\mathbf{x}) & =-\left[\left(\frac{x_{1}+D_{0}}{2}\right)^{2}+\left(\frac{D_{0}-x_{2}}{2}\right)^{2}\right]^{0.5} \leq 0 \\
& =-\left[\left(x_{1}+D_{0}\right)^{2}+\left(D_{0}-x_{2}\right)^{2}\right]^{0.5} \leq 0
\end{aligned}
\end{aligned}
$$

where $x_{1}=\tilde{S}_{0}$ and $x_{2}=\tilde{D}_{1}$. The inequality constraint $g_{2}(\mathbf{x})$ is added to Eq. 14 to ensure that the large diameter cylinders and small diameter cylinders do not overlap.

The governing equations ( 8 to 10 ) were discretised using the Finite Volume Method (FVM) and all internal variable fields were initialised as being 0 (i.e. $\mathbf{U}^{0}=\mathbf{0}, P^{0}=0$ and $T^{0}=0$ ). OpenFoam [27] solves the steady-state Eqs. ( 8 to 10) by introducing a non-zero time derivative (dummy time variable). Thus the steady-state problem is viewed as a transient problem that reaches steady-state at a certain time. The convergence criteria, used to solve the governing equations, at each time step is set based on the residual of each of the variable fields:

$$
R_{t}^{k}(\mathbf{U}) \leq 10^{-4}, \quad R_{t}^{k}(P) \leq 10^{-4}, \quad R_{t}^{k}(T) \leq 10^{-6}
$$

and the convergence criteria to terminate the simulation is evaluated based on the initial residual of each of the variable fields, i.e.:

$$
R_{t}^{0}(\mathbf{U}) \leq 10^{-4}, \quad R_{t}^{0}(P) \leq 10^{-4}, \quad R_{t}^{0}(T) \leq 10^{-6}
$$

In which $k$ is the iteration counter and $t$ is the time step.

The convergence criteria used to terminate the optimisation algorithm was:

$$
\left\|\mathbf{x}^{i}-\mathbf{x}^{i-1}\right\| \leq 10^{-3}
$$


Table 1: Multi scale mesh refinement summary: number of elements per unit length at each Rayleigh number $(\operatorname{Pr}=0.72)$.

\begin{tabular}{lr}
\hline$R a$ & Elements $/ L$ \\
\hline $10^{2}$ & 32 \\
$10^{3}$ & 48 \\
$10^{4}$ & 80 \\
\hline
\end{tabular}

Table 2: Multi scale mesh refinement study for $R a=10^{3}\left(\tilde{H}_{u}=0.5, \tilde{H}_{d}=3.5, \tilde{S}_{0}=0.5\right.$ and $\left.\operatorname{Pr}=0.72\right)$.

\begin{tabular}{lrr}
\hline Elements $/ L$ & $\tilde{q}$ & $\frac{\tilde{q}^{i}-\tilde{q}^{i+1}}{\tilde{q}^{i}}$ \\
\hline 12 & 6.604 & - \\
24 & 6.618 & 0.00219 \\
48 & 6.631 & 0.00199 \\
96 & 6.634 & 0.00041 \\
\hline
\end{tabular}

where $\|\cdot\|$ is the Euclidean norm.

In order to obtain an accurate heat flux results (Eq. 12), from any of the simulations, the computational domain needs to be considered, namely the upstream and downstream lengths as well as the number of elements used to discretise the computational domain. The mesh design received special attention and was tested extensively in the range $10^{2} \leq R a \leq 10^{4}$, for $\tilde{\omega}_{0}=0$ and $\operatorname{Pr}=0.72$, with the grid varying from one geometric configuration to the next. A structured mesh, consisted of hexahedron elements was used. The initial guess for the mesh size in the $\tilde{x}$ direction was chosen based on the boundary layer thickness scale $\delta_{T} \sim D_{0} R a^{(-1 / 4)}$.

Table 1 shows the mesh refinement summary for the number of elements for the multi scale configuration $\left(10^{1} \leq\right.$ $R a \leq 10^{4}$ ). For example the mesh refinement study shows, for $R a=10^{2}$, the heat transfer density rate is insensitive (varies by less than $1 \%$ ) to further mesh refinement when 32 elements per unit length were used. Table 2 shows one example of how mesh independence was achieved for $R a=10^{3}, S_{0}=0.5, D_{1}=0.2$ and $\operatorname{Pr}=0.72$.

Table 3 shows a summary of the domain size study for the upstream and downstream lengths for $\operatorname{Pr}=0.72$. It was found that the heat transfer density rate is insensitive when the downstream length $\tilde{H}_{d}$ scales linearly as a function of the cylinder-to-cylinder spacing minus the small cylinder diameter. For example the domain size study shows for $R a=10^{3}, \tilde{S}_{0}=0.5$, and $\tilde{D}_{1}=0.2$ that the heat transfer density rate is insensitive to further doubling of upstream and downstream lengths when $\tilde{H}_{u}=0.6$ and $\tilde{H}_{d}=-5.11\left(\tilde{S}_{0}-\tilde{D}_{1}\right)+4.95=3.42$.

\section{Numerical Results}

\subsection{Stationary Cylinders}

As discussed above in section 3, an optimisation routine was used to determine the optimal geometric configuration for the maximum heat transfer density rate. The accuracy of results obtained from the optimisation routine need to be considered.

Table 3: Multi scale domain size summary: upstream and downstream lengths at each Rayleigh number $(\operatorname{Pr}=0.72)$.

\begin{tabular}{llc}
\hline$R a$ & $\tilde{H}_{u}$ & $\tilde{H}_{d}=$ \\
\hline $10^{2}$ & 0.9 & $-4.57\left(\tilde{S}_{0}-\tilde{D}_{1}\right)+7.50$ \\
$10^{3}$ & 0.6 & $-5.11\left(\tilde{S}_{0}-\tilde{D}_{1}\right)+4.95$ \\
$10^{4}$ & 0.4 & $-5.31\left(\tilde{S}_{0}-\tilde{D}_{1}\right)+3.15$ \\
\hline
\end{tabular}


Figure 6 shows a contour plot for the heat transfer density rate for a range of cylinder-to-cylinder spacings $\left(\tilde{S}_{0}\right)$ and small cylinder diameters $\left(\tilde{D}_{1}\right)$ for $\operatorname{Ra}=10^{3}, \operatorname{Pr}=0.72$ and $\tilde{\omega}_{0}=0$. The optimum configuration obtained from the optimisation routine is also shown in Fig. 6 (as the "Global optimum") and it can be seen that this result is very feasible and compares well with the data collected. It is also worth mentioning that the heat transfer density rate (objective function) is very well behaved, in the sense that it can be considered to be smooth, continuous and have only one optimum.

The feasibility of the result obtained from the optimisation routine is again shown in Fig. 7. This figure shows that cylinder-to-cylinder spacing can be optimized, for maximum heat transfer density rate, for each small cylinder diameter. It can further be seen, from Fig. 7, that there exists a global optimum heat transfer density rate when $\tilde{S}_{0}$ and $\tilde{D}_{1}$ have a certain value, when there is no cylinder rotation. The global optimum shown in Fig. 7, for $R a=10^{3}$, $\operatorname{Pr}=0.72$ and $\tilde{\omega}_{0}=0$, is found when $\tilde{S}_{0, o p t}=0.557$ and $\tilde{D}_{1, o p t}=0.191$. There is a $14.8 \%$ and $4.5 \%$ difference when comparing this optimal cylinder-to-cylinder spacing and small cylinder diameter, respectively, with that reported by Bello-Ochende and Bejan [12] for a multi scale configuration of cylinders with two length scales.

The optimal cylinder-to-cylinder spacings and corresponding maximum heat transfer density rates for $10^{2} \leq R a \leq$ $10^{4}, \operatorname{Pr}=0.72$ and $\tilde{\omega}_{0}=0$ are summarized in Fig. 8. The optimal cylinder-to-cylinder spacings (from Fig. 8) can be correlated by the power law, within $0.27 \%$ :

$$
\tilde{S}_{0, o p t}=2.113 R a^{(-0.205)}
$$

This correlation compares reasonably well with the power law correlation proposed by Bello-Ochende and Bejan [12]: $\tilde{S}_{0, \text { opt }}=2.218 R a^{(-0.22)}$. For $10^{3} \leq R a \leq 10^{5}$, there is a $5.67 \%$ difference $\left(R a=10^{3}\right), 9.38 \%$ difference $\left(R a=10^{4}\right)$ and $13.24 \%$ difference $\left(R a=10^{5}\right)$.

The corresponding maximum heat transfer density rates, reported in Fig. 8, can be correlated by the power law, within $5.9 \%$ :

$$
\tilde{q}_{m}=0.698 R a^{0.321}
$$

This correlation also compares well with the power law correlation proposed by Bello-Ochende and Bejan [12]: $\tilde{q}_{m}=0.85 R a^{0.30}$. For $10^{3} \leq R a \leq 10^{5}$, there is a $5.06 \%$ difference $\left(R a=10^{3}\right), 0.36 \%$ difference $\left(R a=10^{4}\right)$ and $4.58 \%$ difference $\left(R a=10^{5}\right)$.

\subsection{Rotating Cylinders}

The optimal cylinder-to-cylinder spacings and optimal small cylinder diameters for $10^{2} \leq \operatorname{Ra} \leq 10^{4}, \operatorname{Pr}=0.72$ and $0 \leq \tilde{\omega}_{0} \leq 10$ are summarized in Fig. 9. This figure shows that any cylinder rotation in the range $0 \leq \tilde{\omega}_{0} \leq 1$ has very little improvement on the the optimal geometric configuration for a multi scale structure. There is a maximum decrease in the optimal cylinder-to-cylinder spacing of $3.1 \%, 4.7 \%$ and $0 \%$ for the $R a$ numbers $10^{2}, 10^{3}$ and $10^{4}$ respectively, when the cylinder rotation speed is increased from stationary to $\tilde{\omega}_{0}=1$. It is also shown that, for a cylinder rotation speed of $\tilde{\omega}_{0}=10$, there is a decrease in the optimal cylinder-to-cylinder spacing of $10.74 \%$ and $24.57 \%$ for the $R a$ numbers $10^{2}$ and $10^{3}$ respectively. When the Rayleigh number is equal to $10^{4}$ and the cylinder rotational speed is greater than 1 , the results become non-physical because the laminar model of the flow collapses due to a wake and consequent turbulence, which dominates the flow behind the rotating cylinders.

The optimal small cylinder diameters, shown in Fig. 9, is 0.2 for all cylinder rotation speeds for $R a=10^{4}$ and 0.19 for all cylinder rotation speeds for $R a=10^{3}$ (with the exception of $\tilde{\omega}_{0}=10$ ). This result compares well with the result proposed by Bello-Ochende and Bejan [12]: $\tilde{D}_{1, \text { opt }}=0.2$. For the Rayleigh number $R a=10^{2}$ the optimal small cylinder diameter is 0.1 for all cylinder rotation speeds, this deviation from 0.2 can be attributed to the larger thermal boundary layer around the large cylinder (large slenderness ratio) at this Rayleigh number.

The corresponding maximum heat transfer density rates for $10^{2} \leq \operatorname{Ra} \leq 10^{4}, \operatorname{Pr}=0.72$ and $0 \leq \tilde{\omega}_{0} \leq 10$ are summarized in Fig. 10. From Fig. 10 it can be seen that any cylinder rotation in the range $0 \leq \tilde{\omega}_{0} \leq 1$ has almost no improvement on the the maximum heat transfer density rate for a multi scale structure. There is a maximum increase in heat transfer density rate of $1.2 \%, 1.5 \%$ and $1.2 \%$ for the $R a$ numbers $10^{2}, 10^{3}$ and $10^{4}$ respectively, when the cylinder rotation speed is increased from stationary to $\tilde{\omega}_{0}=1$. It is interesting to note that, at a cylinder rotation speed of $\tilde{\omega}_{0}=10$, the maximum heat transfer density rate is suppressed for all Rayleigh numbers in the range $10^{2} \leq R a \leq 10^{4}$. 
Figure 11 shows a comparison between the multi scale and single scale [29] configurations for the optimal cylinder-to-cylinder spacing. The multi scale configuration has the effect of increases the optimal cylinder-to-cylinder when adding a smaller diameter cylinder in the mouth of the channel. This smaller diameter cylinder also has the effect of restricting the flow through the mouth of the large diameter cylinders and thus cylinder rotation has a minimal effect on the cylinder-to-cylinder spacing. The maximum cylinder packing, from a physical point of view, is obtained using a single scale configuration with rotation.

Figure 12 shows a comparison between the multi scale and single scale [29] configurations for the maximum heat transfer density rate. Although the effect of cylinder rotation (on a single scale configuration) is an increase in the maximum heat transfer density rate, a multi scale configuration (without any cylinder rotation) achieves a higher maximum heat transfer density rate. There is however an exception at very low Rayleigh numbers $\left(R a=10^{1}\right.$ and $R a=10^{2}$ ) where a high cylinder rotation speed increases the maximum heat transfer density rate greater than that of a multi scale configuration.

Figure 13 shows the effect of cylinder rotational speed on the thermal boundary layer for a multi scale structure of rotating cylinders shown in Fig. 1 for $R a=10^{3}$ and $\operatorname{Pr}=0.72$. Figure 13a shows, for $\tilde{\omega}_{0}=0$, that the thermal boundary layer between two consecutive large diameter cylinders, at the optimal geometric configuration, touches just past the top of the cylinders. Figure $13 \mathrm{~b}$ shows, for $\tilde{\omega}_{0}=1$, that the thermal boundary layer between two consecutive large diameter cylinders (where the cylinder rotation aids the flow direction) is extended and touches around one large cylinder diameter past the top of the cylinders. However, unlike the single scale configuration (cf. [29, Fig. 8.]), the thermal boundary layer between two consecutive large diameter cylinders (where the cylinder rotation opposes the flow direction) is more strongly effected and touches near the centreline of the large diameter cylinders.

In summary, the optimal packing for the maximum heat transfer density rate for a given Rayleigh number and cylinder rotation speed (for single scale and multi scale configuration) can be presented in the following equality equation:

$$
\begin{aligned}
\text { if } 10^{1} \leq R a<10^{2}: & \\
\operatorname{dof}= & 1 \\
\tilde{\omega}_{0}= & 10 \\
\tilde{S}_{0, \text { opt }}= & -0.362+1.69 R a^{-0.25} \\
& \text { (cf. Eq. 21, Ref. [29]) } \\
\tilde{q}_{m}= & 0.989+0.71 R a^{0.29} \\
& (\text { cf. Eq. } 22, \text { Ref. [29]) } \\
\text { if } 10^{2}<R a \leq 10^{4}: & \\
\text { dof }= & 2 \\
\tilde{\omega}_{0}= & 0 \\
\tilde{S}_{0, o p t}= & 2.113 R a^{(-0.205)} \\
& (\text { cf. Eq. } 18) \\
\tilde{q}_{m}= & 0.698 R a^{0.321}
\end{aligned}
$$

(cf. Eq. 19)

where dof is the number of degrees of freedom for the flow configuration.

\section{Conclusions}

For a multi scale configuration, the results show that the effect of increasing the rotation of the large diameter cylinders has little to no impact on the heat transfer density rate with the exception of a high rotation speed which serves to suppress the heat transfer density rate. Because the thermal boundary layer between two consecutive large diameter cylinders (where the cylinder rotation opposes the flow direction) is so strongly effected it causes a reduction 
in the heat transfer density rate which effectively cancels out the increase in heat transfer density rate created by the cylinder rotation that aids the flow direction (cf. Fig. 13).

For the maximum heat transfer density rate, the optimal configuration of cylinders was found to be as follows:

- for a Rayleigh number range of $10^{1} \leq R a \leq 10^{2}$, it was found to be beneficial if rotation was added to the cylinders and only a single scale structure was used; and

- for a Rayleigh number range of $10^{2}<R a \leq 10^{4}$, the optimal configuration was one with no cylinder rotation in a multi scale structure. Even though a rotation speed of $\tilde{\omega}_{0}=1$ augmented the heat transfer density rate slightly, it was almost negligible with a maximum increase of around $1.2 \%$, and a higher rotation speed served to suppress the heat transfer density rate.

The optimisation algorithm used in this paper [19] required a lot of objective function evaluations in order to determine the optimal geometric structure. Each objective function evaluation is in effect the solution for one CFD simulation for the given design variable values. A higher number of objective function evaluation leads to more time spent solving for the optimal structure, with the majority of the time being spent solving the CFD simulation. It would therefore be beneficial to research another optimisation algorithm or even develop a new algorithm, specifically tailored for CFD applications, that requires fewer objective function evaluation.

Further research may include a three-dimensional numerical model or to consider the effect of vibration or oscillation of the cylinders on the heat transfer density rate.

\section{Acknowledgements}

The authors acknowledge with gratitude the support from the University of Pretoria and the National Research Foundation (NRF-DST).

\section{References}

[1] H. Badr, S. Dennis, Laminar forced convection from a rotating cylinder, International Journal of Heat and Mass Transfer 28 (1985) 253 264.

[2] A. Bejan, Shape and Structure, from Engineering to Nature, Technical Report, Cambridge University Press, Cambridge, UK, 2000.

[3] A. Bejan, I. Dincer, S. Lorente, A. Miguel, A. Reis, Porous and Complex Flow Structures in Modern Technologies, Springer-Verlag, New York, 1st edition, 2004.

[4] A. Bejan, Y. Fautrelle, Constructal multi-scale structure for maximal heat transfer density, Acta Mechanica 163 (2003) 39 - 49.

[5] A. Bejan, S. Lorente, The constructal law and the thermodynamics of flow systems with configuration, International Journal of Heat and Mass Transfer 47 (2004) $3203-3214$.

[6] A. Bejan, S. Lorente, Design with Constructal Theory, John Wiley \& Sons, Inc., 1st edition, 2008.

[7] A. Bejan, A. Morega, The optimal spacing of a stack of plates cooled by turbulent forced convection, International Journal of Heat and Mass Transfer 37 (1994) 1045 - 1048.

[8] A. Bejan, E. Sciubba, The optimal spacing for parallel plates cooled by forced convection, International Journal of Heat and Mass Transfer 35 (1992) $3259-3264$.

[9] T. Bello-Ochende, A. Bejan, Maximal heat transfer density: plates with multiple lengths in forced convection, International Journal of Thermal Sciences 43 (2004) 1181 - 1186.

[10] T. Bello-Ochende, A. Bejan, Optimal spacings for mixed convection, Journal of Heat Transfer 126 (2004) 956 - 962

[11] T. Bello-Ochende, A. Bejan, Constructal multi-scale cylinders in cross-flow, International Journal of Heat and Mass Transfer 48 (2005) 1373 -1383 .

[12] T. Bello-Ochende, A. Bejan, Constructal multi-scale cylinders with natural convection, International Journal of Heat and Mass Transfer 48 (2005) $4300-4306$.

[13] T. Bello-Ochende, J. Meyer, J. Dirker, Three-dimensional multi-scale plate assembly for maximum heat transfer rate density, International Journal of Heat and Mass Transfer 53 (2010) 586 - 593.

[14] T. Bello-Ochende, J. Meyer, O. Ogunronbi, Constructal multiscale cylinders rotating in cross-flow, International Journal of Heat and Mass Transfer 54 (2011) 2568 - 2577.

[15] C. Chiou, S. Lee, Forced convection on a rotating cylinder with an incident air jet, International Journal of Heat and Mass Transfer 36 (1993) $3841-3850$.

[16] B. Gschaider, Contrib PyFoam, http://openfoamwiki.net/index.php/Contrib_PyFoam, 2011. [Online; accessed 5-December-2011].

[17] M. Gshwendtner, Optical investigation of the heat transfer from a rotating cylinder in a cross flow, Heat and Mass Transfer 40 (2004) 561 572 .

[18] R. Issa, Solution of the implicitly discretised fluid flow equations by operator-splitting, Journal of Computational Physics 62 (1986) 40 - 65. 
[19] E. Jones, T. Oliphant, P. Peterson, Others, SciPy: Open source scientific tools for Python, http://www .scipy . org/, 2011. [Online; accessed 14-November-2011]

[20] M. Joucaviel, L. Gosselin, T. Bello-Ochende, Maximum heat transfer density with rotating cylinders aligned in cross-flow, International Communications in Heat and Mass Transfer 35 (2008) 557 - 564.

[21] D. Kraft, Algorithm 733: Tomp - 8211; fortran modules for optimal control calculations, ACM Trans. Math. Softw. 20 (1994) $262-281$.

[22] C. Lawson, H. R.J., Solving Least Square Problems, Prentice-Hall, Englewood Cliffs, New Jersey, 1974.

[23] A. Mohanty, A. Tawfek, B. Prasad, Heat transfer from a rotating cylinder in crossflow, Experimental Thermal and Fluid Science 10 (1995) $54-61$.

[24] M. Nobari, K. Gharali, M. Tajdari, A numerical study of flow and heat transfer in internally finned rotating curved pipes, Numerical Heat Transfer; Part A: Applications 56 (2009) 76 - 95.

[25] M. Oesterle, M. Lauster, R. Waibel, V. Lippig, D. Straub, Topological structures near a heated rotating cylinders, Experiments in Fluids 24 (1998) $308-322$

[26] OpenCFD Ltd, OpenFOAM: The open source cfd toolbox, http://www . openf oam. com, 2011. [Online; accessed 11-October-2011].

[27] OpenCFD Ltd, OpenFOAM: User guide, http: //www . openfoam. com/docs/user/, 2011. [Online; accessed 11-October-2011].

[28] B. Ozerdem, Measurement of convective heat transfer coefficient for a horizontal cylinder rotating in quiescent air, International Communications in Heat and Mass Transfer 27 (2000) 389 - 395.

[29] L. Page, T. Bello-Ochende, J. Meyer, Maximum heat transfer density rate enhancement from cylinders rotating in natural convection, International Communications in Heat and Mass Transfer 38 (2011) 1354 - 1359.

[30] S. Panda, R. Chhabra, Laminar forced convection heat transfer from a rotating cylinder to power-law fluids, Numerical Heat Transfer; Part A: Applications 59 (2011) $297-319$.

[31] S. Paramane, A. Sharma, Numerical investigation of heat and fluid flow across a rotating circular cylinder maintained at constant temperature in 2-d laminar flow regime, International Journal of Heat and Mass Transfer 52 (2009) $3205-3216$.

[32] S. Paramane, A. Sharma, Heat and fluid flow across a rotating cylinder dissipating uniform heat flux in $2 \mathrm{~d}$ laminar flow regime, International Journal of Heat and Mass Transfer 53 (2010) 4672 - 4683.

[33] Python Software Foundation, Python programming language - official website, http://www.python.org/, 2011. [Online; accessed 19October-2011].

[34] A. da Silva, A. Bejan, Constuctal multi-scale structure for maximal heat transfer density in natural convection, International Journal of Heat and Fluid Flow 26 (2005) $34-44$

[35] A. da Silva, A. Bejan, S. Lorente, Maximal heat transfer density in vertical morphing channels with natural convection, Numerical Heat Transfer Part A - Applications 45 (2004) 135 - 152.

[36] G. Stanescu, A. Fowler, A. Bejan, The optimal spacing between horizontal cylinders in a fixed volume cooled by natural convection, International Journal of Heat and Mass Transfer 38 (1995) 2047 - 2055.

[37] G. Stanescu, A. Fowler, A. Bejan, The optimal spacing of cylinders in free-stream cross-flow forced convection, International Journal of Heat and Mass Transfer 39 (1996) 311 - 317

[38] Y. Yan, Y. Zu, Numerical simulation of heat transfer and fluid flow past a rotating isothermal cylinder — a lbm approach, International Journal of Heat and Mass Transfer 51 (2008) 2519 - 2536 


\section{Figure Captions:}

Fig. 1. Single row of counter-rotating cylinders in natural convection.

Fig. 2. Illustration of the thermal boundary layer around a stationary cylinder.

Fig. 2a. Single scale configuration.

Fig. 2b. Multi scale configuration.

Fig. 3. The computational domain and boundary conditions for a set of counter-rotating cylinders.

Fig. 4. Parametrisation routine for OpenFoam.

Fig. 5. Optimisation routine for OpenFoam.

Fig. 6. Contour plot of the heat transfer density rates for a row of rotating cylinders shown in Fig. 1 for $R a=10^{3}$ and $P r=0.71$.

Fig. 7. The maximum heat transfer density rates for a row of rotating cylinders shown in Fig. 1 for $R a=10^{3}$ and $\operatorname{Pr}=0.71$.

Fig. 8. The optimal cylinder-to-cylinder spacings and corresponding heat transfer density rates for a row of cylinders shown in Fig. 1 for $\operatorname{Pr}=0.72, \tilde{\omega}_{0}=0$ and $10^{2} \leq \operatorname{Ra} \leq 10^{4}$.

Fig. 9. The optimal cylinder-to-cylinder spacings and small cylinder diameter for a row of rotating cylinders shown in Fig. 1 for $P r=0.72$.

Fig. 10. The maximum heat transfer density rates for a row of rotating cylinders shown in Fig. 1 for $P r=0.72$.

Fig. 11. The effect of increasing of cylinder rotational speed and the effect of increasing the complexity on the optimal cylinder-to-cylinder spacing for $\operatorname{Pr}=0.72$.

Fig. 12. The effect of increasing of cylinder rotational speed and the effect of increasing the complexity on the maximum heat transfer density rate for $\mathrm{Pr}=0.72$.

Fig. 13. The effect of cylinder rotational speed on the thermal boundary layer for a multi scale structure of rotating cylinders shown in Fig. 1.

Fig. 13a. $\tilde{\omega}_{0}=0$

Fig. 13b. $\tilde{\omega}_{0}=1$ 


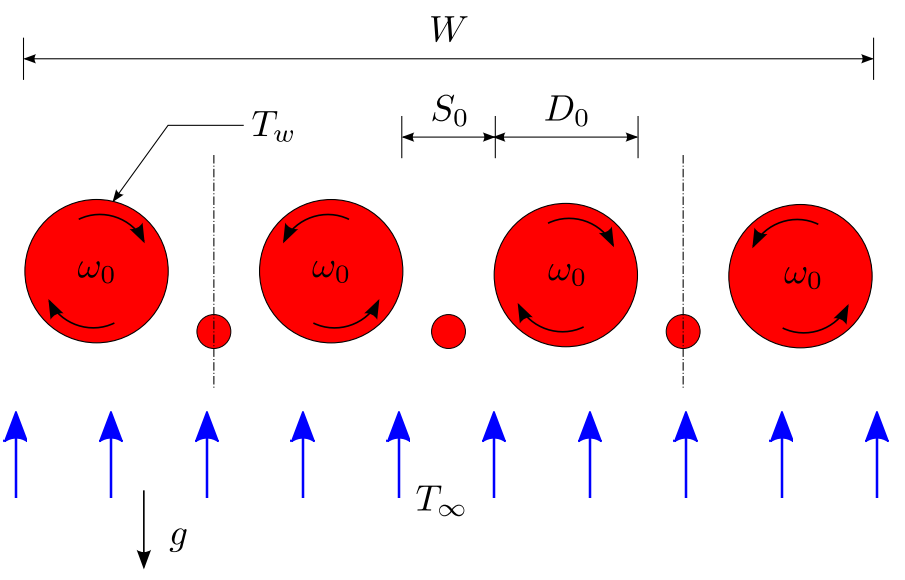

Figure 1

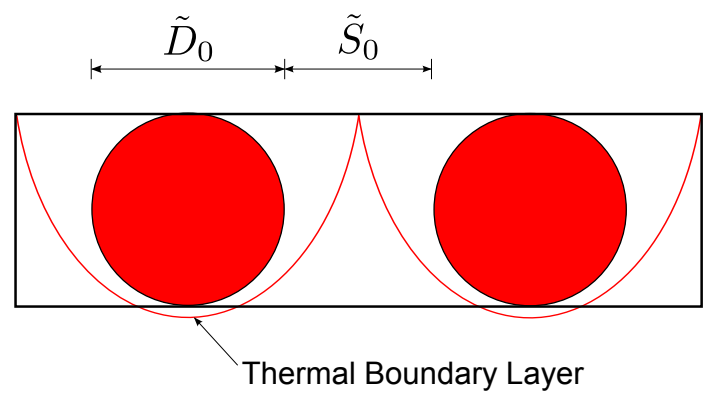

(a)

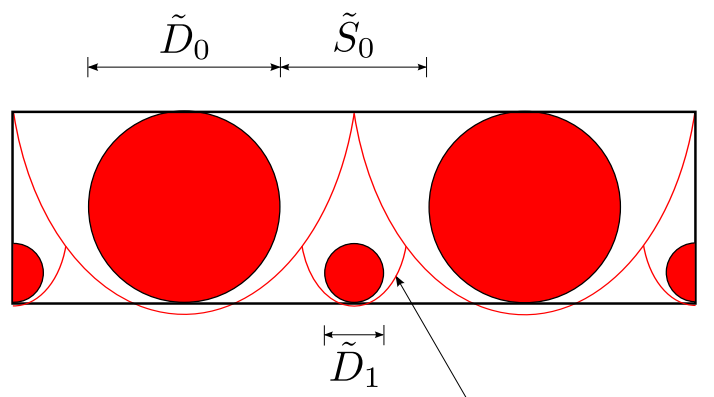

Thermal Boundary Layer

(b)

Figure 2 


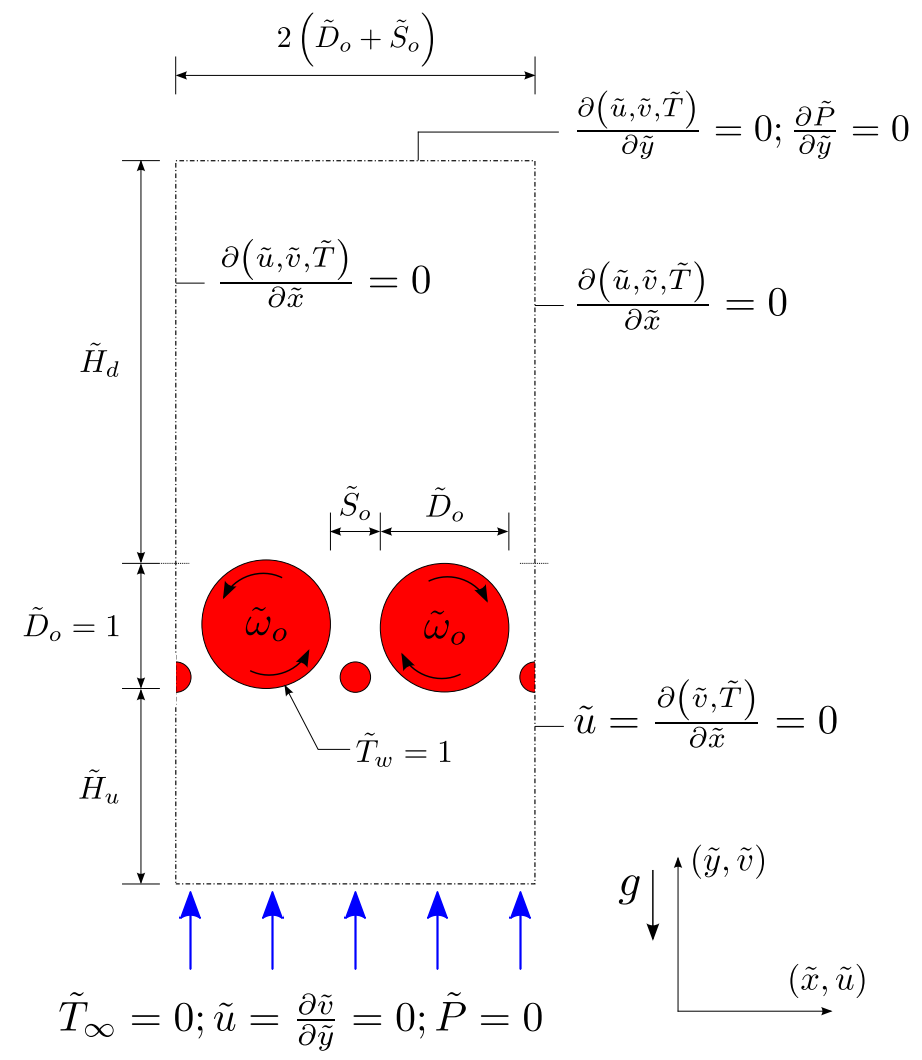

Figure 3 


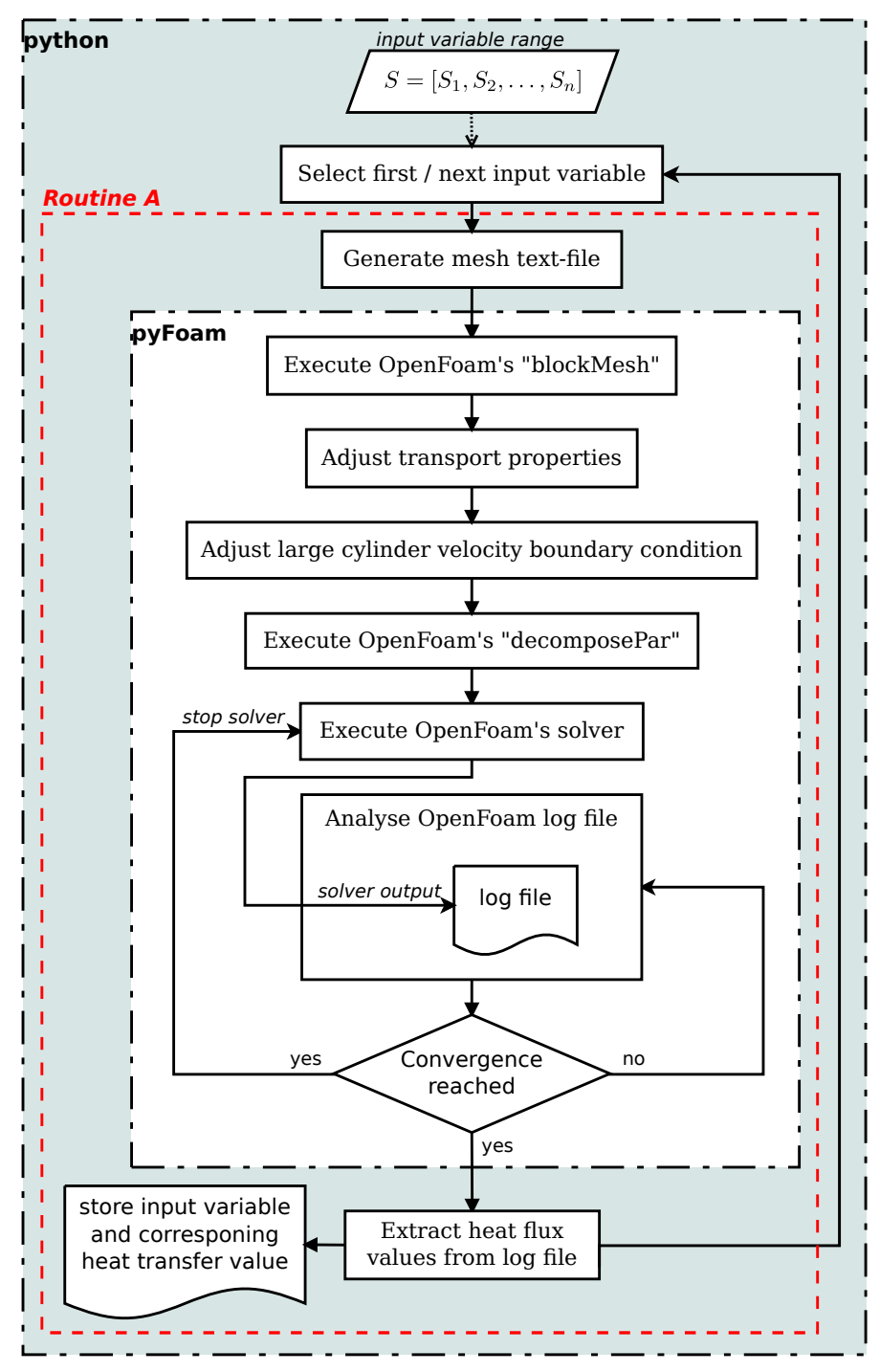

Figure 4 


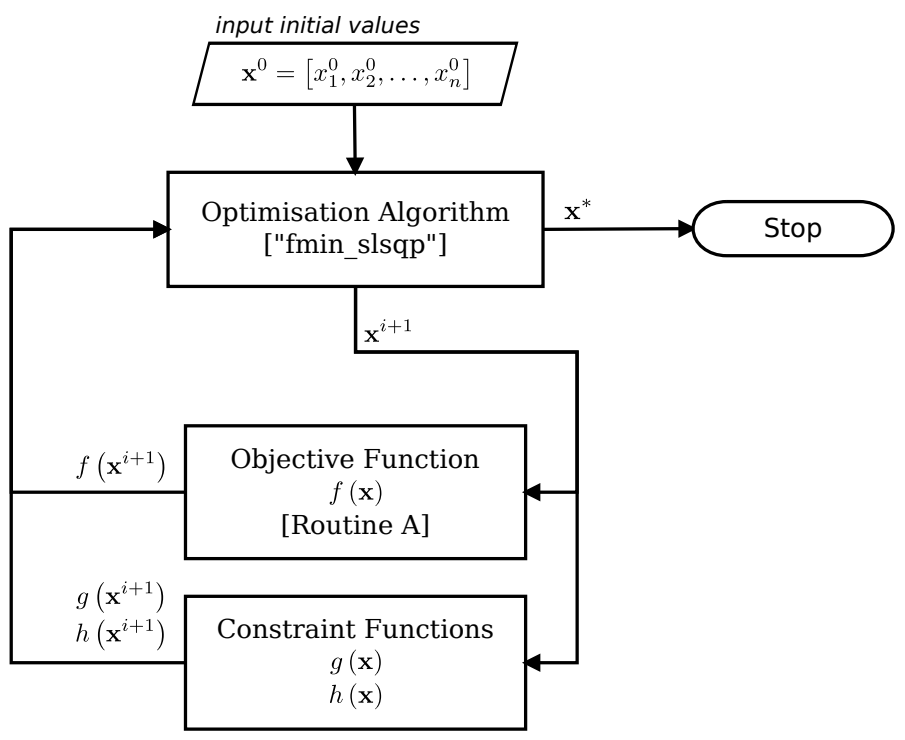

Figure 5

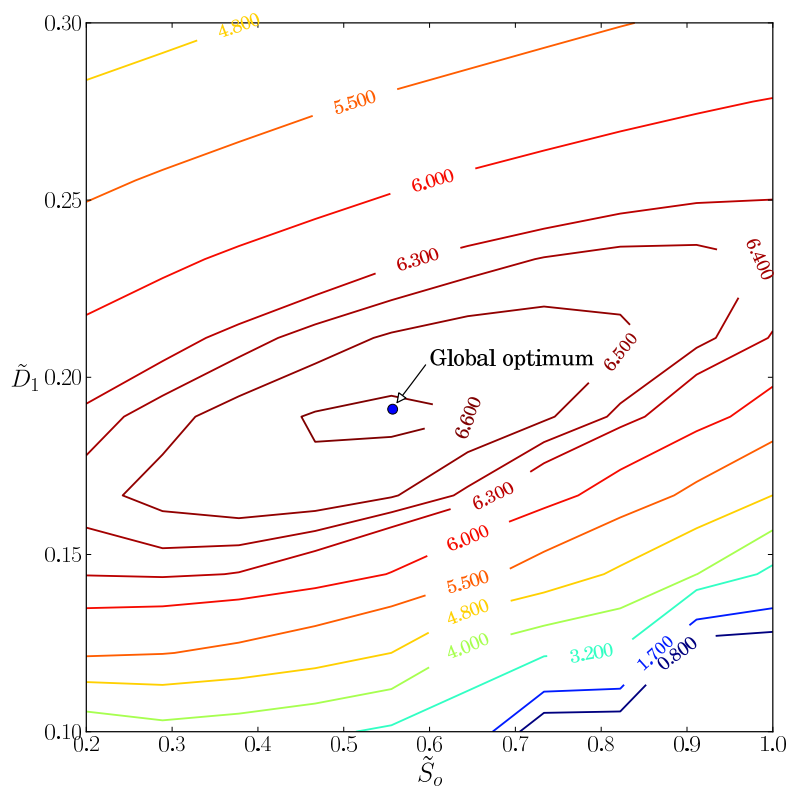

Figure 6 


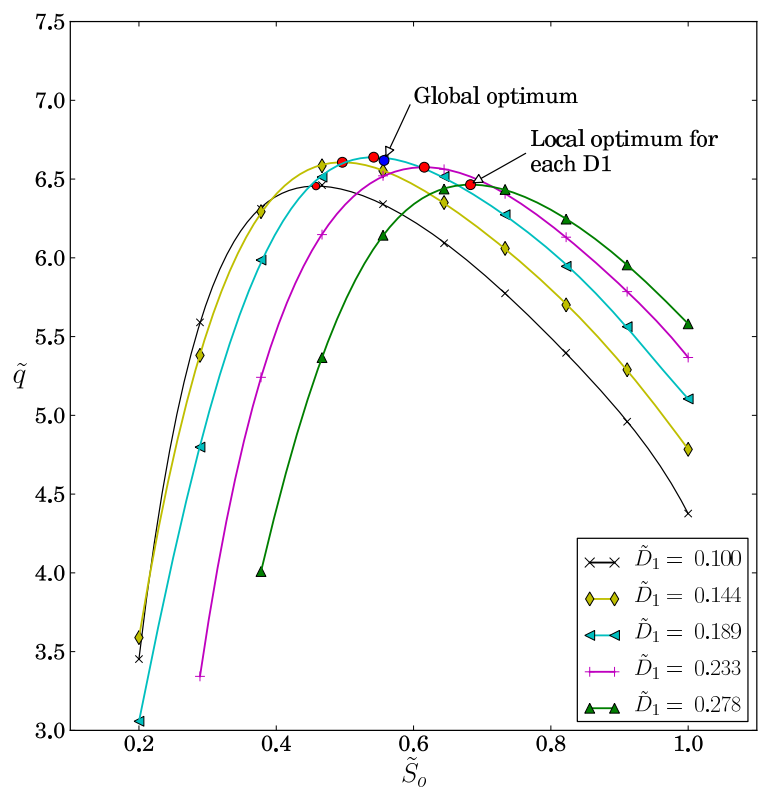

Figure 7

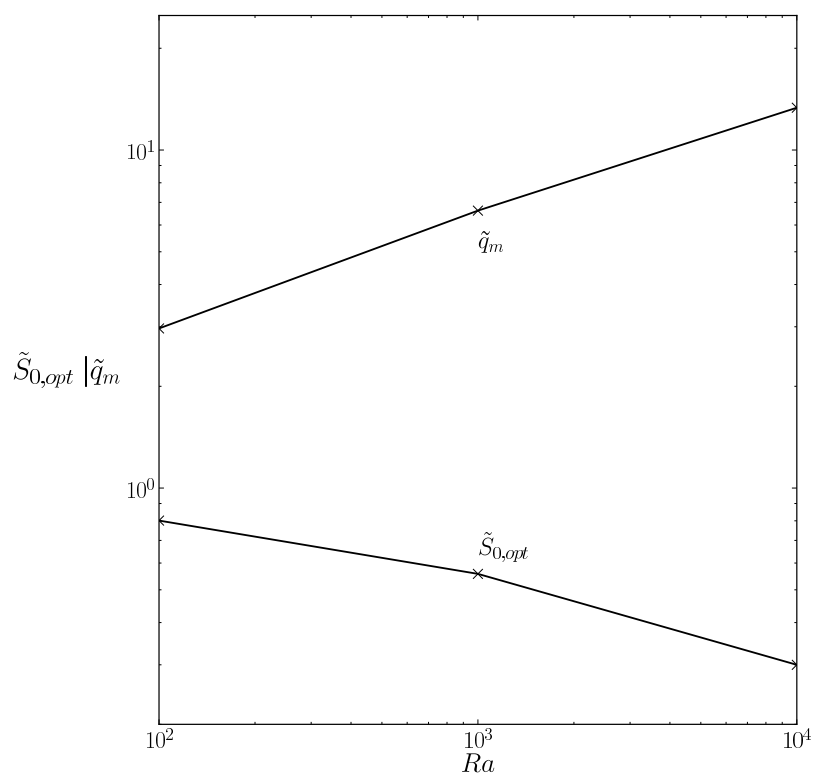

Figure 8 


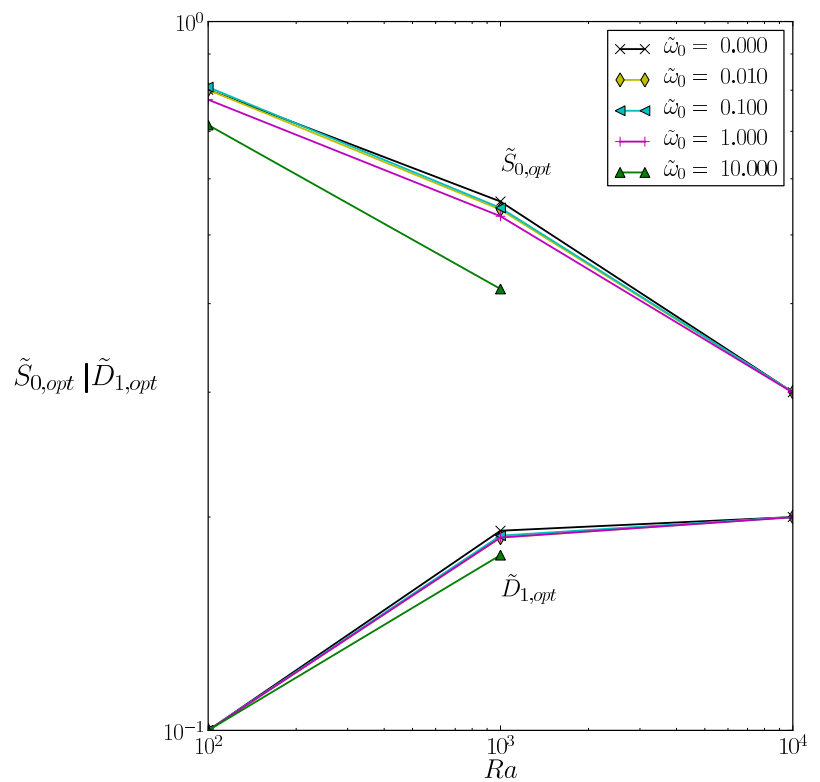

Figure 9

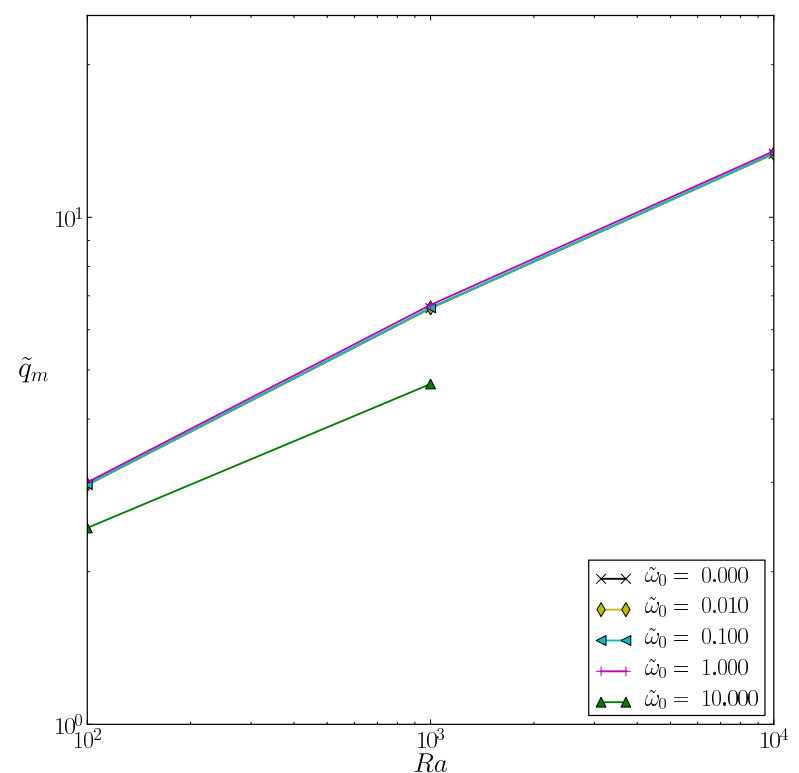

Figure 10 


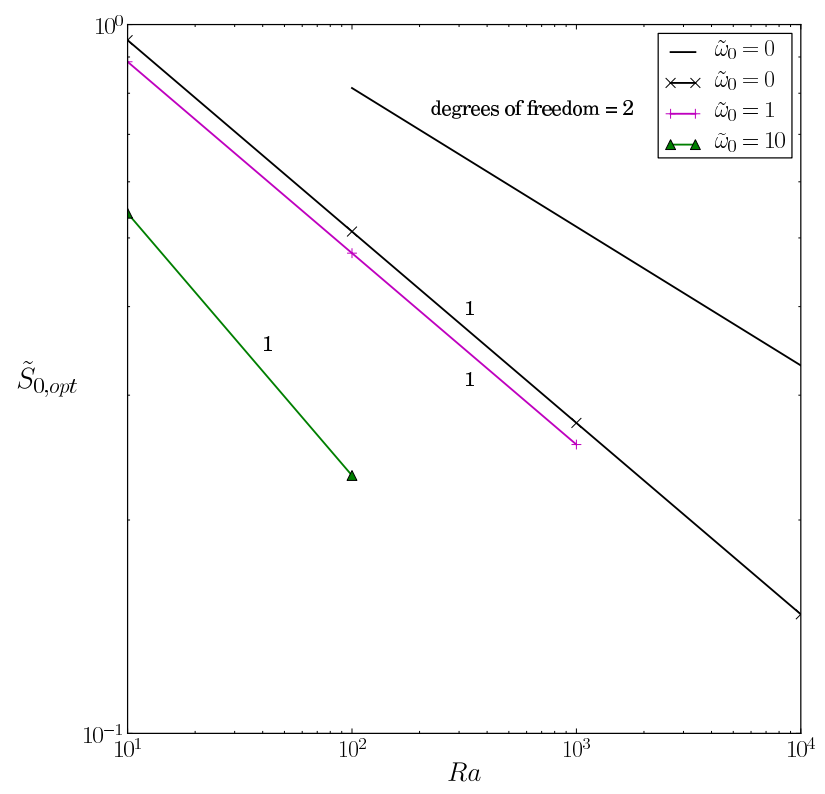

Figure 11

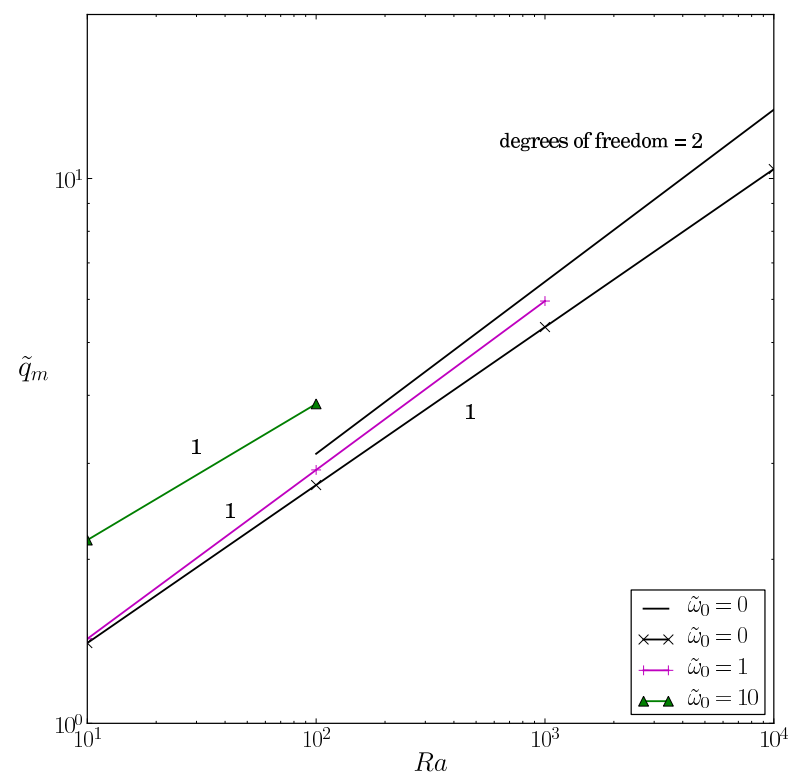

Figure 12 


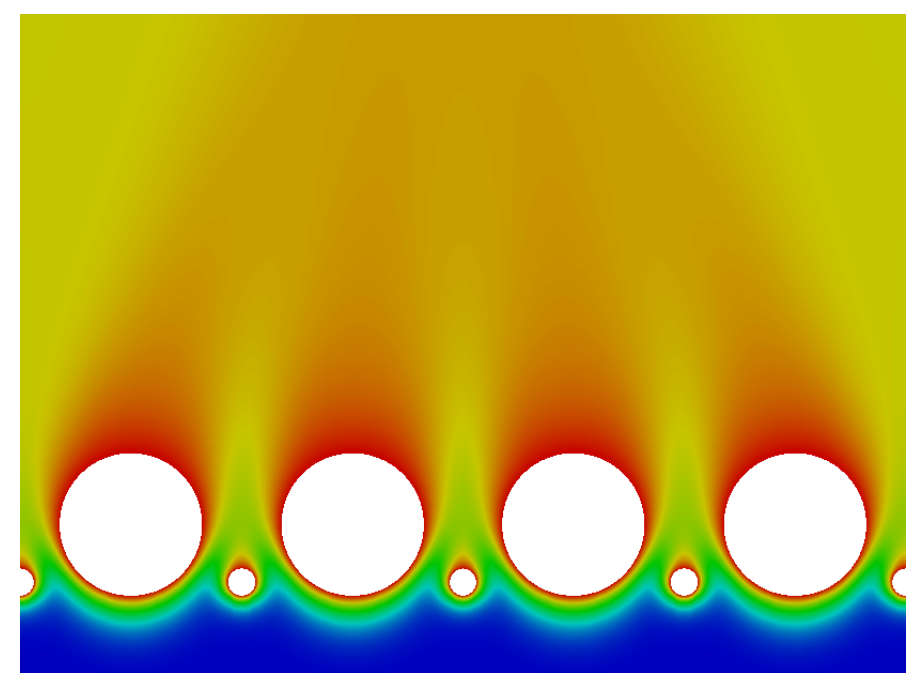

(a)

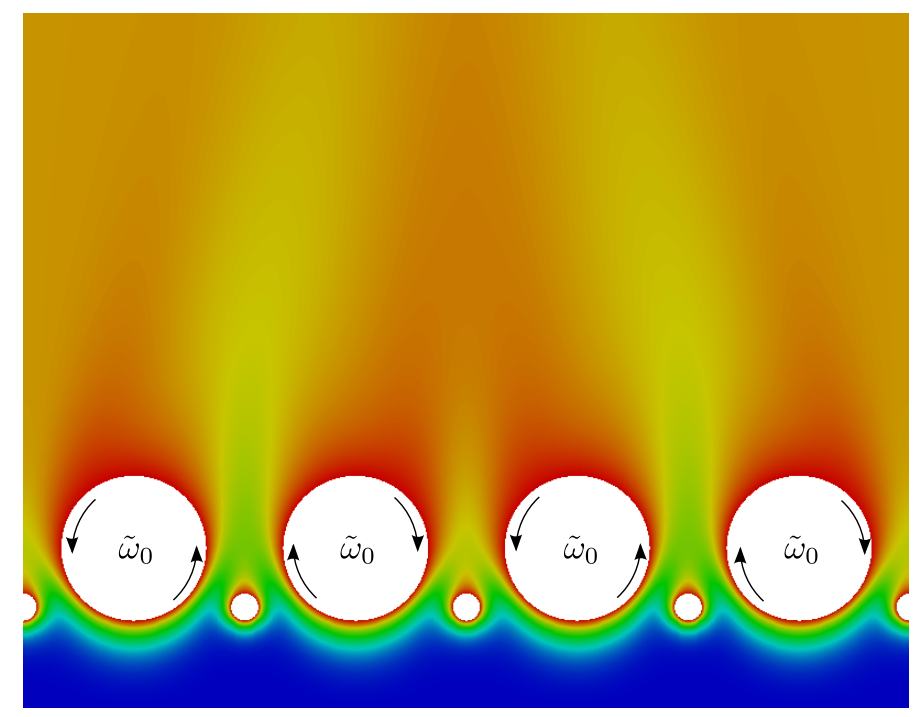

(b)

Figure 13 